\title{
Observation of highly flexible residues in amyloid fibrils of the HET-s prion.
}

Ansgar B. Siemer, Alexandre A. Arnold, Christiane Ritter, Thomas Westfeld, Matthias Ernst, Roland Riek and Beat H. Meier

\section{Supplementary Material}

Chemical shifts in of the tentatively assigned amino acids in [ppm]. Chemical shifts were referenced relative to DSS.

Ala: $\delta C^{\alpha}=52.6, \delta C^{\beta}=19.1, \delta H^{\alpha}=4.32, \delta H^{\beta}=1.39$

Arg: $\delta C^{\beta}=30.7, \delta C^{\gamma}=27.0, \delta C^{\delta}=43.4, \delta H^{\beta}=1.77 / 1.87, \delta H^{\gamma}=1.63, \delta H^{\delta}=3.20$

Asn/Asp: $\delta C^{\beta}=39.4, \delta H^{\beta}=2.74$

Asn/Asp: $\delta C^{\beta}=41.1, \delta H^{\beta}=2.59 / 2.72$

Gln: $\delta C^{\beta}=30.0, \delta C^{\gamma}=33.8, \delta H^{\beta 2 / 3}=2.11 / 2.04, \delta H^{\gamma}=2.35$

Glu: $\delta C^{\alpha}=56.3, \delta C^{\beta}=31.7, \delta C^{\gamma}=36.2, \delta H^{\beta 2 / 3}=1.94 / 2.07, \delta H^{\gamma}=2.27$

His: $\delta C^{\beta}=29.7, \delta C^{\delta 2}=119.8, \delta C^{\varepsilon 1}=136.4, \delta H^{\beta}=3.19 / 3.09, \delta H^{\delta 2}=7.18, \delta H^{\varepsilon 1}=8.46$

Ile: $\delta C^{\beta}=38.7, \delta C^{\gamma 1}=27.3, \delta C^{\gamma 2}=17.5, \delta C^{\delta}=13.0, \delta H^{\beta}=1.88, \delta H^{\gamma 12 / 13}=1.18 / 1.46, \delta H^{\gamma 2}=0.89$, $\delta H^{\delta}=0.85$

Leu: $\delta C^{\delta 1 / 2}=23.4 / 24.8, \delta H^{\delta 1 / 2}=0.84 / 0.89$

Lys: $\delta C^{\beta}=33.1, \delta C^{\gamma}=24.7, \delta C^{\delta}=29.1, \delta C^{\varepsilon}=42.1, \delta H^{\beta}=1.74 / 1.83, \delta H^{\gamma}=1.41, \delta H^{\delta}=1.67, \delta H^{\varepsilon}=$ 2.99

Met: $\delta C^{\gamma}=30,8, \delta C^{\varepsilon}=16.8, \delta H^{\gamma}=2.56, \delta H^{\varepsilon}=2.09$

Thr: $\delta C^{\gamma}=21.8, \delta H^{\beta}=4.22, \delta H^{\gamma}=1.20$

Val: $\delta C^{\alpha}=62.4, \delta C^{\beta}=32.7, \delta C^{\gamma 1 / 2}=20.7 / 21.0, \delta H^{\alpha}=4.10, \delta H^{\beta}=2.07, \delta H^{\gamma}=0.94 / 0.92$ 
Comparison of the measured chemical shifts with its random-coil, $\beta$-strand and $\alpha$-helical values*.

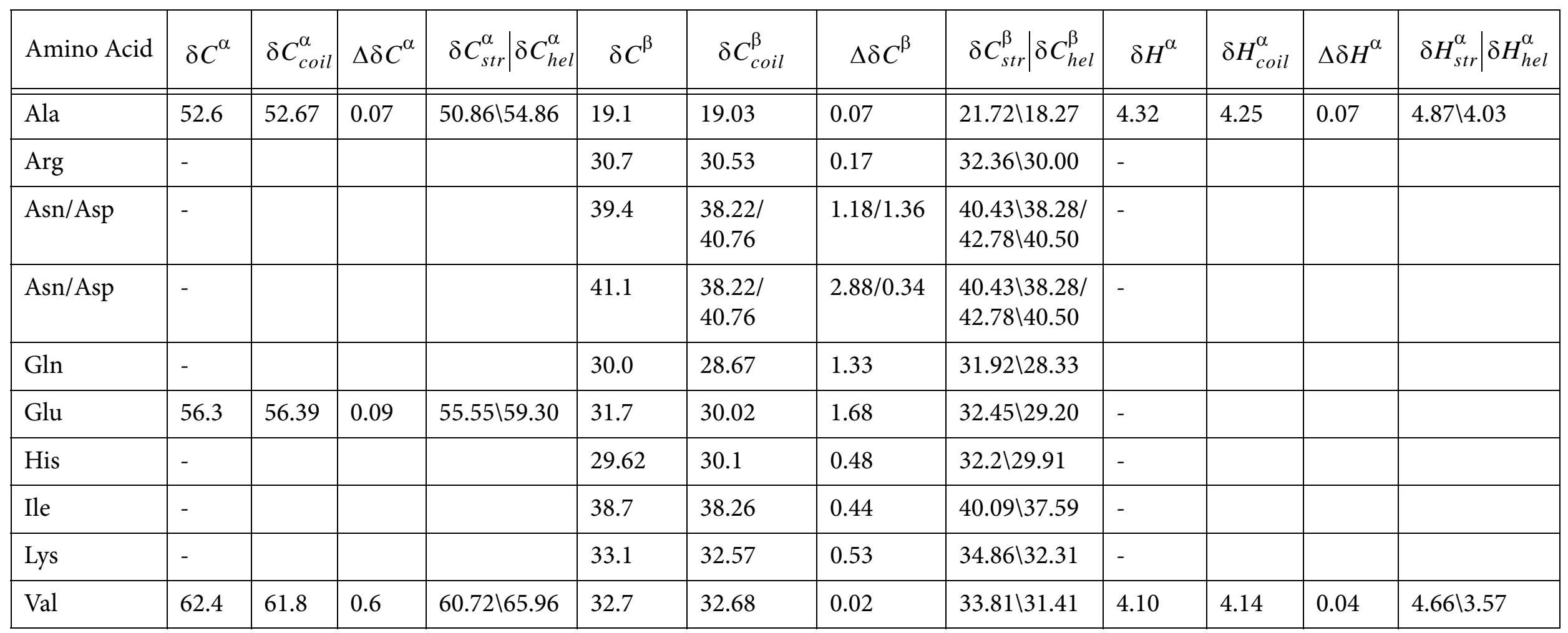

*average random-coil, $\beta$-strand and $\alpha$-helical values were taken from Y. Wang, O. Jardetzky, Prot. Sci. 2001, 11, 852-861 